\title{
Sensitivity and Specificity of Two WHO Approved SARS-CoV2 Antigen Assays in Detecting Patients with SARS-CoV2 Infection
}

\section{Chandima Jeewandara}

University of Sri Jayewardenepura Faculty of Medical Sciences

\section{Dinuka Guruge}

Colombo Municipal Council

\section{Pradeep Pushpakumara}

University of Sri Jayewardenepura Faculty of Medical Sciences

Deshan Madushanka

University of Sri Jayewardenepura Faculty of Medical Sciences

Tibutius Jayadas

University of Sri Jayewardenepura Faculty of Medical Sciences

Indika Chathuranga

Colombo Municipal Council

\section{Inoka Abeyratne}

University of Sri Jayewardenepura Faculty of Medical Sciences

\section{Saubhagya Danasekara}

University of Sri Jayewardenepura Faculty of Medical Sciences

Thilagaraj Pathmanathan

University of Sri Jayewardenepura Faculty of Medical Sciences

Deshni Jayathilaka

University of Sri Jayewardenepura Faculty of Medical Sciences

\section{Gayasha Somathilaka}

University of Sri Jayewardenepura Faculty of Medical Sciences

\section{Heshan Kuruppu}

University of Sri Jayewardenepura Faculty of Medical Sciences

\section{Laksiri Gomes}

University of Sri Jayewardenepura Faculty of Medical Sciences

Vijith Gunasekara

Ministry of Health, Sri Lanka

\section{Ruwan Wijayamuni}

Colombo Municipal Council

Graham Ogg 
University of Oxford

Gathsaurie Neelika Malavige ( $\square$ gathsaurie.malavige@ndm.ox.ac.uk)

University of Sri Jayewardenepura https://orcid.org/0000-0001-9201-0449

\section{Research article}

Keywords: SARS-CoV-2, Rapid antigen tests, PCR, Abbott Ag test, SD-Biosensor Ag test, antibodies

Posted Date: June 16th, 2021

DOl: https://doi.org/10.21203/rs.3.rs-618824/v1

License: (c) (i) This work is licensed under a Creative Commons Attribution 4.0 International License. Read Full License

Version of Record: A version of this preprint was published at BMC Infectious Diseases on March 22nd, 2022. See the published version at https://doi.org/10.1186/s12879-022-07240-6. 


\section{Abstract}

Background: SARS-CoV-2 rapid antigen (Ag) detection kits are widely used in addition to quantitative realtime PCR (RT-qPCR), as they are cheaper with a rapid turnaround time. As there are many concerns regarding their sensitivity and specificity, in different settings, we evaluated two WHO approved rapid Ag kits in a large cohort of Sri Lankan individuals.

Methods: Paired nasopharangeal swabs were obtained from 4845 participants for validation of the SDBiosensor rapid Ag assay and 3625 for the Abbott rapid Ag assay, in comparison to RT-qPCR. A short questionnaire was used to record symptoms at the time of testing, and blood samples were obtained from 2721 of them for detection of SARS-CoV-2 specific antibodies.

Results: The overall sensitivity of the SD-Biosensor Ag kit was 36.5\% and the Abbott Ag test was $50.76 \%$. The Abbott Ag test showed specificity of $99.4 \%$ and the SD-Biosensor Ag test $97.5 \%$. At Ct values $<25$, the sensitivity was $71.3 \%$ to $76.6 \%$ for the SD-Biosensor Ag test and $77.3 \%$ to $88.9 \%$ for the Abbott Ag test. The Ct values for all genes (RdRP, S, E and N) tested with all RT-qPCR kits were significantly lower for the positive results of the Abbott Ag test compared to the SD-Biosensor test. 209 (48.04\%) individuals who had antibodies gave a positive RT-qPCR result, and antibody positivity rates were higher at Ct values $>30$ (46.1 to $82.9 \%$ ). $32.1 \%$ of those who gave a positive result with the SD-Biosensor Ag test and $26.3 \%$ of those who gave positive results with the Abbott Ag test had SARS-CoV-2 antibodies at the time of detection.

Conclusions: Both rapid Ag tests appeared to be highly sensitive in detecting individuals at lower $\mathrm{Ct}$ values, in a community setting in Sri Lanka, but it will be important to further establish the relationship to infectivity.

\section{Background}

Quantitative real time PCR (RT-qPCR), with use of specific primers targeting two or three genes of the SARS-CoV2 virus is considered as the gold-standard in the diagnosis of a patient infected with the virus $[1,2]$. However, the use of RT-qPCR has several limitations in relation to poor sensitivity and also due to the difficulties in using these in community surveillance [3]. Due to the high cost, the need of dedicated equipment and trained individuals, use of RT-qPCR has been challenging for many developing countries with scare resources. In order to fulfill this need many diagnostic tests which are faster and cheaper, such the loop-mediated isothermal amplification (RT-LAMP) for detection of viral RNA[4], CRISPR-based assays [5] and many different types of lateral flow antigen detection assays $[1,6,7]$.

The rapid antigen assays (RATs) are convenient to use due to the very fast turnaround time, to able to test the individuals at the point of care and also as they are relatively cheaper. However, there have been many concerns regarding their poor sensitivity and sometimes the occurrence of false-positive results $[7,8]$. While these RATs appear to be the ideal test in community surveillance programs, their pretest probability depends on the prevalence of SARS-CoV2 in the community [7]. The pre-test probability is lower in 
communities with lower prevalence rates giving rise to higher false positive rates $[7,8]$. The World Health Organization recommends a minimum of $80 \%$ sensitivity and $97 \%$ specificity in the RATs that are used for diagnostic purposes[8]. However, the studies that have assessed the sensitivity and specificity of the currently approved RATSs, have varied in study design and in some have been evaluated in a relatively small number of individuals [9]. Furthermore, it has been reported that certain SARS-CoV-2 can given to false negative results due to certain mutations that occur in the $\mathrm{N}$ protein [10].

Although the sensitivity of these RATs have been questioned, they are thought to be adequately sensitive to detect individuals with higher viral loads, especially during the early phases of infection and therefore, who are likely to be more infectious [9]. Therefore, the WHO has recommended their use to investigate certain outbreak situations, to monitor disease trends in communities and for early detection and isolation of infected individuals in setting where there is a high degree of community transmission [9]. Although the use of these RATs are not recommended in setting where there is a low community prevalence to test individuals especially before elective procedures such as elective surgery, blood donation and at points of entry [9], many countries use the tests in these setting due to the costs and time associated with the PCRs. As the performance of these RATs is known to vary in different settings, with different SARS-CoV-2 variants, we proceeded to evaluate the performance of two of the test kits, in a large cohort in individuals in the city of Colombo, Sri Lanka.

\section{Methods}

\section{Participants}

The Colombo Municipality Council (CMC) has a population of 752,993 individuals in an area of $37.3 \mathrm{~km}^{2}$ and is one of the highest population dense areas $\left(20,187.5\right.$ persons $\left./ \mathrm{km}^{2}\right)$ in the country. The highest number of cases of COVID-19 were reported in the CMC area during October 2020 to mid-January 2021, which reported, 13216 cases during this period. Therefore, in order to identify cases in the community, RTqPCR was carried out in primary contacts of cases and also during community surveillance. Since the SD-Biosensor and Abbott RATs were first used in Sri Lanka since end of November, individuals residing in the $\mathrm{CMC}$ were invited to participate in this study. Paired nasopharyngeal swabs for each participant was obtained for the RAT and RT-qPCR. A short questionnaire was used to record symptoms at the time of testing, and self-reported symptoms of fever, sore throat, cough and diarrhoea were recorded. In 2721 of the participants, a blood sample was also obtained for the detection of SARS-CoV-2 antibodies, following informed written consent.

Informed consent and human experimentation guidelines

Informed written was obtained from all participants, in whom a blood sample was collected. Ethics approval for the study was obtained from the Ethics Review Committee of the University of Sri Jayewardenepura.

\section{Realtime RT-PCR for detection of SARS CoV-2:}


Nasopharyngeal swabs samples of the participants were lysed and RNA was extracted using QIAmp Viral RNA Mini Kit (Qiagen, USA, Cat: 52906) and used to detect the presence of the SARS-CoV2 virus using four different PCR kits; Allplex ${ }^{\text {TM }}$ SARS-CoV-2 Assay (SeeGene, Korea), TaqPath ${ }^{\text {TM }}$ COVID-19 RT-PCR Kit (Thermo Fisher Scientific, USA), STANDARD M nCoV Real-Time Detection kit (SD Biosensor,Korea) and COVID-19 Real-time PCR Kit (HBRT-COVID-19) (Chaozhou Hybribio Biochemistry Ltd, China) as different kits were available in our laboratory during different time periods. The qRT-PCR carried out according to the manufacturer's instructions. A cycle threshold value (Ct) of $\leq 38$ for two or more genes was considered as a positive PCR result.

\section{SD Biosensor and Abbott RAT}

Nasopharyngeal swabs samples of 4845 participants were used for validation of the SD-Biosensor RAT and 3625 for the Abbott RAT. Dedicated sterilized nasopharyngeal swabs in the kit were used to collect the sample. Samples for the Abbott PanbioTM COVID-19 RAT (Abbott, Germany) and the Standard Q covid 19 Ag test (SD Biosensor, Korea) were collected, and the assay carried out and interpreted according to the manufacturers instructions. All the samples were processed within an hour of the sample collection.

\section{Detection of antibodies to SARS-CoV-2}

SARS-COV-2 specific antibodies (IgM, IgG and IgA) were assessed using WANTAI SARS-CoV-2 Ab ELISA (Beijing Wantai Biological Pharmacy Enterprise, China) in 2721 individuals in blood samples obtained at the time paired samples were obtained for RT-qPCR/RAT. The Wantai ELISA assay was shown to have a sensitivity of $98 \%$ [11] and was found to be $100 \%$ specific in serum samples obtained in 2018, in Sri Lankan individuals. The assay was carried out and results were interpreted according to manufacturers' instructions.

\section{Statistical analysis}

GraphPad Prism version 6 was used for statistical analysis. As the data were not normally distributed, differences in means were compared using the Mann-Whitney $U$ test (two tailed). The descriptive statistics including the frequencies, percentages with $95 \%$ confidence intervals, cross-tabulations, measures of sensitivity, specificity, positive and negative predictive values were calculated using the MS Excel and R software tool (R version 3.3.3 (2017-03-06) and R-Studio Version 1.3.959).

\section{Results}

\section{Performance of the SD-Biosensor RAT}

In order to evaluate the sensitivity and specificity of the SD-Biosensor RAT, paired samples were tested in 4845 individuals. The sensitivity and specificity of the SD-Biosensor RAT was evaluated against three PCR kits; SeeGene $(n=2427)$, Taqpath $(n=1667)$ and SD Biosensor $(n=692)$, as different kits were available in our laboratory during different time periods. All three of these kits have the RdRP gene of the 
SARS-CoV2 as a target, while SeeGene has $\mathrm{N}$ and $\mathrm{E}$ genes as the other target genes, Taqpath has $\mathrm{N}$ and $\mathrm{S}$ as the other genes and SD-Biosensor E gene as the other target gene.

Of the 4845 individuals, 893 gave a positive RT-qPCR result, 3925 were negative and 27 gave an inconclusive result. Of the 893 RT-qPCR positive individuals, the RAT was positive in 324 (36.3\%) individuals, negative in 567 (63.5\%) and invalid result in 2 individuals. Therefore, the overall sensitivity of the SD-Biosensor RAT was $36.4 \%$ (95\% Cl-33.3-39.8\%). Of those who had a negative RT-qPCR result, 96/3925 (2.4\%) gave a positive RAT result and therefore, the specificity was $97.7 \%(95 \% \mathrm{Cl}-97-98 \%)$. The positive predictive value (PPV) of the assay compared to RT-qPCR was $76.5 \%$ and the negative predictive value (NPV), was $87.6 \%$. The sensitivity of this RAT at different Ct values for different PCR kits is shown in Table 1.

Table 1

Positivity rate of the SD-Biosensor rapid Ag kit for different RT-qPCR kits at different Ct values

\begin{tabular}{|lllll|}
\hline PCR Kit & $\begin{array}{l}\text { Avg Ct Value } \\
\text { for PCR }\end{array}$ & $\begin{array}{l}\text { Sensitivity of the SD- } \\
\text { Biosensor Ag kit }\end{array}$ & $\begin{array}{l}95 \% \text { Cl Lower } \\
\text { Limit }\end{array}$ & $\begin{array}{l}95 \% \text { CI Upper } \\
\text { Limit }\end{array}$ \\
\hline SeeGene Kit & $<25$ & $76.6 \%$ & $69.5 \%$ & $82.5 \%$ \\
\cline { 2 - 3 } & $26-30$ & $36.2 \%$ & $28.1 \%$ & $45.1 \%$ \\
\hline Kit & $31-38$ & $6.1 \%$ & $3.3 \%$ & $10.6 \%$ \\
\hline $\begin{array}{l}\text { SD-Biosensor } \\
\text { RT-qPCR }\end{array}$ & 25 & $71.3 \%$ & $60.4 \%$ & $80.2 \%$ \\
Kit & $26-30$ & $13 \%$ & $6.7 \%$ & $23 \%$ \\
\hline
\end{tabular}

The viral load in a respiratory sample inversely correlates with the cycle threshold (Ct). As individuals with lower Ct values, have higher viral loads and likely to be more infectious [12], we evaluated the sensitivity of this RAT at different Ct values of the RdRp gene, E gene, $\mathrm{N}$ gene and $\mathrm{S}$ that was used in the RT-qPCR assay (Fig. 1). For the RdRp gene, the median Ct value of SD-Biosensor RAT positive samples was 26 (IQR 23 to 40) by the SeeGene kit, 25 (IQR 19 to 40) by the Taqpath kit and 10 (IQR 10 to 23) by the SDBiosensor RT-qPCR kit (Fig. 1A). For the RAT negative samples, the median Ct values for all three kits was 40 (IQR 40 to 40). The S gene target was only present in the Taqpath kit and the median Ct values for RAT positive samples was 26 (IQR 19.75 to 40) (Fig. 1B). Two PCR kits had primers targeting the $\mathrm{N}$ gene and the median Ct value of SD-Biosensor RAT positive samples was 17 (IQR 16 to 20) for the SeeGene kit and 26 (IQR 22 to 39) by the Taqpath kit and 26 (IQR 20 to 40) (Fig. 1C). Two kits had primers targeting the $E$ gene and the median Ct value of SD-Biosensor Ag positive samples in the SeeGene kit was 24 (IQR 19 to 35) and for the SD-Biosensor RT-qPCR kit it was 19 (IQR11 to 20) (Fig. 1D). 


\section{Performance of the Abbot RAT}

For determining the sensitivity and specificity of the Abbott SARS-CoV2 RAT, the RAT and RT-qPCR were carried out in 3625 individuals. The sensitivity and specificity of the Abbott RAT were evaluated against three PCR kits; SeeGene $(n=555)$, Taqpath $(n=2490)$ and Hyrbribio $(n=280)$, as different kits were available in our laboratory during different time periods. Of the 3625 individuals, 327 gave a positive RTqPCR result, 3288 were negative and 10 gave an inconclusive result. Of the 327 RT-qPCR positive individuals, the RAT was positive in 166 (50.76\%) individuals and negative in 161(49.24\%). Of those who had a negative RT-qPCR result, 20/3288 gave a positive RAT result. Therefore, the overall sensitivity of the RAT was $50.76 \%(95 \% \mathrm{Cl}-45.2-56.3 \%)$ and the specificity was $99.4 \%(95 \% \mathrm{Cl}-99.0-99.6 \%)$. The PPV of the assay compared to RT-qPCR was $89.2 \%$ and the NPV was $95.3 \%$. The sensitivity of this RAT at different $\mathrm{Ct}$ values for different PCR kits is shown in Table 2.

Table 2

Positivity rate of the Abbott rapid Ag kit for different RT-qPCR kits at different Ct value

\begin{tabular}{|c|c|c|c|c|}
\hline PCR Kit & $\begin{array}{l}\text { Avg Ct Value for } \\
\text { PCR }\end{array}$ & $\begin{array}{l}\text { Sensitivity of the Abbott } \\
\text { Ag kit }\end{array}$ & $\begin{array}{l}95 \% \text { Cl Lower } \\
\text { Limit }\end{array}$ & $\begin{array}{l}\text { 95\% Cl Upper } \\
\text { Limit }\end{array}$ \\
\hline \multirow{3}{*}{$\begin{array}{l}\text { SeeGene } \\
\text { Kit }\end{array}$} & $<25$ & $77.3 \%$ & $54.2 \%$ & $91.3 \%$ \\
\hline & $26-30$ & $7.1 \%$ & $0.4 \%$ & $35.8 \%$ \\
\hline & $31-38$ & $0 \%$ & $0 \%$ & $22.9 \%$ \\
\hline \multirow{3}{*}{$\begin{array}{l}\text { TAQPATH } \\
\text { Kit }\end{array}$} & $<25$ & $81.4 \%$ & $72.9 \%$ & $87.7 \%$ \\
\hline & $26-30$ & $18.7 \%$ & $10.9 \%$ & $29.7 \%$ \\
\hline & $31-38$ & $25 \%$ & $4.5 \%$ & $64.4 \%$ \\
\hline \multirow{3}{*}{$\begin{array}{l}\text { Hybri-Bio } \\
\text { Kit }\end{array}$} & $<25$ & $88.9 \%$ & $63.9 \%$ & $98.1 \%$ \\
\hline & $26-30$ & $60 \%$ & $17 \%$ & $92.7 \%$ \\
\hline & $31-38$ & $11.1 \%$ & $0.6 \%$ & $49 \%$ \\
\hline
\end{tabular}

The sensitivity and specificity of this RAT was also evaluated for different Ct values of the RdRp gene, E gene, $\mathrm{N}$ gene and $\mathrm{S}$ that was used in the RT-qPCR assay (Fig. 2). For the RdRp gene, the median Ct value of Abbott RAT positive samples was 17 (IQR 14 to 18.25) for the SeeGene kit, 20.5 (IQR 18 to 24) by the Taqpath kit and 22 (IQR 19.25 to 24.75) by the Hybribio kit (Fig. 2A). For the RAT negative samples, the median $\mathrm{Ct}$ values for all three kits was 40 (IQR 40 to 40). For the $S$ gene, which was only targeted by the Taqpath kit, the median Ct value of RAT positive samples was 22 (IQR 18 to 26) (Fig. 2B). All three PCR kits had primers targeting the $\mathrm{N}$ gene and the median $\mathrm{Ct}$ value of Abbott RAT positive samples was 17 (IQR 16 to 20) for the SeeGene kit, 21 (IQR 18 to 26) by the Taqpath kit and 23 (IQR 21 to 26) by the Hybribio kit (Fig. 2C). The SeeGene kit only had primers targeting the E gene and the median Ct value of RAT positive samples was 16.5 (IQR 14 to 18) (Fig. 2D). 


\section{Comparison of the Ct values in the samples which gave a positive result by the SD-Biosensor vs the Abbot RAT}

In order to determine the differences in detection by the two RATs at different Ct values, we compared the Ct values of the samples which gave a positive result for the SD-Biosensor RAT and the Abbott RAT for the RdRP, S, N and the E genes. This comparison was only carried out for the SeeGene and the Taqpath kits as the SD-Biosensor RT-qPCR kits were not used to validate the Abbott RAT and the Hybribio PCR kit was not used to validate the SD-Biosensor RAT. For RdRP, S, N and the E gene, the Ct values of the Abbott RAT positive samples was significantly less for the SD-Biosensor RAT positive samples for both PCR kits (Fig. 3). For the RdRP gene the Ct values of the Abbot RAT positive samples was significantly less for the SeeGene kit $(p<0.0001)$ than the Taqpath kit $(p=0.001)(F i g .3 A)$. The Ct values of the Abbott RAT positives were again significantly lower for the $S$ gene with the Taqpath kit $(p=0.0007)$ (Fig. 3B) and also for the $N$ gene in the SeeGene kit $(p<0.0001)$ and the Taqpath kit $(p<0.0001)$ (Fig. 3C). The Ct values of the Abbott RAT positives were again significantly lower for the $S$ gene with the Taqpath kit $(p=0.0007)$ (Fig. 3B) and also for the $\mathrm{N}$ gene in the SeeGene kit $(p<0.0001)$ and the Taqpath kit $(p<0.0001)$ (Fig. 3C). Only the Seegene kit targeted the $\mathrm{E}$ gene and again the $\mathrm{Ct}$ values of the Abbott RAT positives were again significantly lower in the Abbott RAT positive samples compared to the SD-Biosensor Ag positive samples $(p<0.0001)$ (Fig. 3D).

\section{Presence of SARS-CoV2 antibodies and the performance of the RATs}

Individuals infected with the SARS-CoV2 virus may shed virus for a prolonged period [13, 14], but are often thought to shed dead, or non-culturable virus $[15,16]$. The appearance of neutralizing antibodies are thought to associate with the presence of non-culturable and therefore, virus particles that are noninfectious [15]. Therefore, it has been proposed that seroconversion and $\mathrm{Ct}$ values of $>24$ could be used to release individuals from isolation/quarantine [15]. As one of the most important uses of the SARSCoV2 RATs it to detect people who are infectious, we proceeded to investigate their performance in individuals from the community and in those who are primary and secondary contacts of the index patient. We obtained blood samples from 2721 individuals at the same time as paired nasopharyngeal samples were obtained for RT-qPCR and antigen testing. The presence of SARS-CoV2 specific antibodies were detected using the Wantai ELISA, which detects the presence of virus specific $\lg A$, IgM and $\lg G$ antibodies and was shown to have a sensitivity of $98 \%$ [11]. The specificity of this assay was evaluated in the Sri Lankan population in 81 serum samples obtained in 2018 and was found to be $100 \%$ specific.

Of the study participants, 435 (15.98\%), were found to have SARS-CoV-2 specific antibodies, although they were not aware of any recent or past COVID-19 illness. 209 (48.04\%) individuals who had antibodies gave a positive RT-qPCR result, while 226 (52.4\%) SARS-CoV2 antibody positive individuals were negative for RT-qPCR. The presence of SARS-CoV2 antibodies for the three different PCR kits, at different Ct values is shown in Table 3. 
Table 3

The presence of SARS-CoV2 specific antibodies for different RT-qPCR kits and different Ct values

\begin{tabular}{|llll|}
\hline Avg Ct Values & Seegene & TAQPATH & SD-Biosensor \\
\hline$<25$ & $21 / 80=26.25 \%$ & $15 / 63=23.81 \%$ & $10 / 24=41.67 \%$ \\
\hline $26-30$ & $41 / 52=78.85 \%$ & $22 / 62=35.48 \%$ & $12 / 17=70.59 \%$ \\
\hline $31-38$ & $58 / 70=82.86 \%$ & $6 / 12=50 \%$ & $18 / 39=46.15 \%$ \\
\hline
\end{tabular}

Of those who had a positive result with the SD-Biosensor RAT 60/187 (32.1\%) had detectable SARS-CoV2 antibodies, while antibodies were detected in 432/1751 (24.6\%) of those who tested negative result (Table 4). Of those who tested positive with the Abbott RAT 10/38 (26.3\%) were also positive for antibodies, and 109/602 (18.1\%) who tested negative for the Abbott RAT, had SARS-CoV2 specific antibodies.

Table 4

The presence of SARS-CoV2 specific antibodies, in those who were tested positive vs negative for the SARS-CoV2 rapid antigen kits

\begin{tabular}{|llll|}
\hline & Ab Positive & Ab Negative & Total \\
\hline SD-Biosensor Ag Positive & $60(32.1 \%)$ & 127 & 187 \\
\hline SD-Biosensor Ag Negative & $432(24.6 \%)$ & 1319 & 1751 \\
\hline Abbott Ag Positive & $10(26.3 \%)$ & 28 & 38 \\
\hline Abbott Ag Negative & $109(18.1 \%)$ & 493 & 602 \\
\hline
\end{tabular}

\section{Disease symptoms and sensitivity of RAT compared to RT-qPCRs}

Four symptoms were recorded at the time of testing in all participants and if they reported at least one of the four symptoms (fever, sore throat, cough or diarrhea) they were considered to be symptomatic. Of the 8470 individuals, 249 (2.94\%) were symptomatic and 8221 (97.1\%) were asymptomatic at the time of testing. Of those who were asymptomatic 1115 (13.2\%) were PCR positive. Of those who were PCR positive, 847 (75.9\%) were positive by the SD-Biosensor and 268 (24.03\%) by the Abbott RAT. Of those who were asymptomatic and PCR negative, 88/7106 (1.23\%) tested positive for the SD RAT and 20/7106 $(0.28 \%)$ tested positive for the Abbott RAT.

209 (48.4\%) of the PCR positive individuals had SARS-CoV-2 antibodies at the time of testing. 8 of them were symptomatic while 201 (96.2\%) were asymptomatic. While numbers of RT-qPCR positive, antibodypositive symptomatic individuals is small as expected, there was no significant difference in RT-qPCR positivity and presence or absence of symptoms or antibody positivity.

\section{Discussion}


In this study we have evaluated the performance of two WHO approved RATs in a large cohort of individuals $(\mathrm{n}=8470)$ in an area of high prevalence $(1.76 \%)$ when the dominant circulating variant was a virus of the SARS-CoV-2 B.1.411 lineage[17]. In Sri Lanka, the control strategy consisted of identifying every possible infected individual through testing of all contacts of cases and also through community screening in locations where increased numbers of SARS-CoV2 infected individuals were identified. Therefore, it was important to evaluate if RAT were a good tool to identify infected individuals, in the community, in comparison to RT-qPCR. Compared to RT-qPCR, the overall sensitivity of the SD-Biosensor RAT was $36.5 \%$ and the Abbott RAT was $50.76 \%$. The Abbott RAT was also found to be more specific $(99.4 \%)$ than the SD-Biosensor RAT (97.5\%). Therefore, in an area of a high level of transmission, $96 / 3925=2.4 \%$ individuals who were identified as being negative by RT-qPCR were given a positive result by the SD-Biosensor RAT, whereas the false positivity rates of the Abbott RAT were lower (20/3288 = 0.6\%). The PPV and the NPV were also significantly better in the Abbott RAT (89.2\% and $95.3 \%)$ compared to the SD-Biosensor RAT (76.5\% and 87.6\%). The Abbott RAT indicated positives at significantly lower $\mathrm{Ct}$ values for all genes for all the RT-qPCR kits tested compared to the SD-Biosensor RAT. As the Abbott RAT gives a positive result at lower Ct values, it is less likely to give a false positive result and therefore, has a high specificity, PPV and an NPV.

Although patients who are infected with the SARS-CoV-2 virus have the highest viral loads during early illness, which associates with infectivity, they can shed non-infectious virus for many weeks $[18,19]$. Lower Ct values ( $<20)$ have shown to associate with infectivity [20,21] and also associate with a higher probability of culturing the virus [22]. Therefore, the RATs that detect patients having higher viral loads (lower Ct values), are thought to be ideal in identifying individuals who are most infectious and likely to spread the virus. Indeed, the Abbot RAT gave positive results for samples with a median Ct value of 17 to 23 for different genes and different PCR kits, while the SD RAT gave positive results for samples with a median $\mathrm{Ct}$ value between 18 to 26 for different gene targets and kits. Therefore, these RAT do appear to be useful in identifying patients with higher viral loads, who are possibly more infectious.

However, it has been established that individuals with mild or asymptomatic infection are unlikely to be infectious 7 to 10 days since the onset of symptoms [16, 23]. SARS-CoV-2 specific antibodies become detectable after 7 days since onset of illness and sometimes much later in patients with asymptomatic of mild illness $[13,24]$. In our cohort of patients, $97.1 \%$ were tested positive were asymptomatic at the time of testing. $48.04 \%$ of these individuals had antibodies, suggesting that they had been harboring the infection for some time. Therefore, a large proportion of asymptomatic individuals who are tested positive by RT-qPCR and thus isolated from the community seem likely to have passed their infective period. Interestingly, of those who had Ct values of $<25,23.8-41.67 \%$ had detectable antibodies. This suggests that even those with lower $\mathrm{Ct}$ values at time of detection are likely to have had the infection for a while and shedding non-infectious virus particles. Therefore, low Ct values per se at the time of detection in asymptomatic individuals, does not seem to indicate that the person is in the early phase of the illness. However, antibody positivity was higher with $\mathrm{Ct}$ values $>30$, with the antibody positivity ranging from $82.86 \%$ for the SeeGene kit to $46.15 \%$ for the SD-Biosensor PCR assay. 
$32.1 \%$ of those who gave a positive result with the SD-Biosensor RAT and $26.3 \%$ of those who were positive with the Abbott RAT, had SARS-CoV-2 antibodies at the time of testing. Therefore, RAT do not appear to necessarily detect only infectious individuals, as a significant proportion of those who tested positive by RATs seem to have had the infection for at least more than 7 days.

One of the main limitations of this study is that the performance of these RATs was evaluated in identification of infection of primary and secondary contacts of cases and in community surveillance. As the majority of this cohort of individuals were asymptomatic when the predominant SARS-CoV2 variant was B.1.411[17], these RATs could give different sensitivity and specificity in individuals who are symptomatic, in a hospital setting. Furthermore, if these assays were evaluated in a location of high prevalence and again the sensitivity and specificity, PPV and NPV could be different in areas where there is a low prevalence of SARS-CoV2 infection.

\section{Declarations}

\section{Ethics approval and consent to participate}

Ethics approval for the study was obtained from the Ethics Review Committee of the University of Sri Jayewardenepura.

\section{Consent for publication}

Not applicable.

\section{Availability of data and materials}

All data are included in the manuscript and figures.

\section{Competing interests}

No conflicts of interests.

\section{Funding}

We are grateful to the World Health Organization, the UK Medical Research Council for their support and the Foreign and Commonwealth Office.

\section{Authors' contributions}

CJ, DG, GNM: Conceptualization

CJ, DG, PD, DJ, LS: Methodology

DG, GNM, GS: Formal analysis 
PD, DM, TTJ, IPC, ISA, SD, TP, DJ, HK, LG: Investigation

DG, DJ, ISA SD, RW: Data curation

CJ, DG, RW, VG : Project administration

CJ, GNM, GSO: Funding acquisition

GNM, CJ, GS: Writing original draft

GNM, GSO, CJ: Writing-review and editing

\section{Acknowledgements}

Not applicable.

\section{References}

1. Tang YW, Schmitz JE, Persing DH, Stratton CW. Laboratory Diagnosis of COVID-19: Current Issues and Challenges. Journal of clinical microbiology 2020, 58(6).

2. Kevadiya BD, Machhi J, Herskovitz J, Oleynikov MD, Blomberg WR, Bajwa N, Soni D, Das S, Hasan M, Patel M, et al: Diagnostics for SARS-CoV-2 infections. Nat Mater 2021.

3. Mina MJ, Peto TE, Garcia-Finana M, Semple MG, Buchan IE: Clarifying the evidence on SARS-CoV-2 antigen rapid tests in public health responses to COVID-19. Lancet 2021.

4. Yoshikawa R, Abe H, Igasaki Y, Negishi S, Goto H, Yasuda J. Development and evaluation of a rapid and simple diagnostic assay for COVID-19 based on loop-mediated isothermal amplification. PLoS Negl Trop Dis. 2020;14(11):e0008855.

5. Broughton JP, Deng X, Yu G, Fasching CL, Servellita V, Singh J, Miao X, Streithorst JA, Granados A, Sotomayor-Gonzalez A, et al. CRISPR-Cas12-based detection of SARS-CoV-2. Nature biotechnology. 2020;38(7):870-4.

6. Chaimayo C, Kaewnaphan B, Tanlieng N, Athipanyasilp N, Sirijatuphat R, Chayakulkeeree M, Angkasekwinai N, Sutthent R, Puangpunngam N, Tharmviboonsri T, et al. Rapid SARS-CoV-2 antigen detection assay in comparison with real-time RT-PCR assay for laboratory diagnosis of COVID-19 in Thailand. Virol J. 2020;17(1):177.

7. Centre for Disease Control U. Interim Guidance for Antigen Testing for SARS-CoV-2. In. National Center for Immunization and Respiratory Diseases (NCIRD), Division of Viral Diseases; 2020.

8. Peeling RW, Olliaro PL, Boeras DI, Fongwen N: Scaling up COVID-19 rapid antigen tests: promises and challenges. The Lancet infectious diseases 2021.

9. WHO: Antigen-detection in the diagnosis of SARS-CoV-2 infection using rapid immunoassays. In: Interim guidance. Emergencies Preparedness, WHO Headquarters (HQ): Emergencies Preparedness, WHO Headquarters (HQ); 2020: 9. 
10. FDA. Genetic Variants of SARS-CoV-2 May Lead to False Negative Results with Molecular Tests for Detection of SARS-CoV-2 - Letter to Clinical Laboratory Staff and Health Care Providers. In.: FDA; 2021.

11. Weidner L, Gansdorfer S, Unterweger S, Weseslindtner L, Drexler C, Farcet M, Witt V, Schistal E, Schlenke P, Kreil TR, et al. Quantification of SARS-CoV-2 antibodies with eight commercially available immunoassays. J Clin Virol. 2020;129:104540.

12. Singanayagam A, Patel M, Charlett A, Lopez Bernal J, Saliba V, Ellis J, Ladhani S, Zambon M, Gopal R. Duration of infectiousness and correlation with RT-PCR cycle threshold values in cases of COVID19, England, January to May 2020. Euro Surveill 2020, 25(32).

13. Jeewandara C, Jayathilaka D, Gomes L, Wijewickrama A, Narangoda E, Idampitiya D, Guruge D, Wijayamuni R, Manilgama S, Ogg GS, et al. SARS-CoV-2 neutralizing antibodies in patients with varying severity of acute COVID-19 illness. Sci Rep. 2021;11(1):2062.

14. Cevik M, Tate M, Lloyd O, Maraolo AE, Schafers J, Ho A. SARS-CoV-2, SARS-CoV, and MERS-CoV viral load dynamics, duration of viral shedding, and infectiousness: a systematic review and metaanalysis. Lancet Microbe. 2021;2(1):e13-22.

15. van Kampen JJA, van de Vijver D, Fraaij PLA, Haagmans BL, Lamers MM, Okba N, van den Akker JPC, Endeman H, Gommers D, Cornelissen JJ, et al. Duration and key determinants of infectious virus shedding in hospitalized patients with coronavirus disease-2019 (COVID-19). Nat Commun. 2021;12(1):267.

16. Perera R, Tso E, Tsang OTY, Tsang DNC, Fung K, Leung YWY, Chin AWH, Chu DKW, Cheng SMS, Poon LLM, et al. SARS-CoV-2 Virus Culture and Subgenomic RNA for Respiratory Specimens from Patients with Mild Coronavirus Disease. Emerg Infect Dis. 2020;26(11):2701-4.

17. Jeewandara C, Jayathilaka D, Ranasinghe D, Hsu NS, Ariyaratne D, Jayadas TT, Madushanka D, Lindsey BB, Gomes L, Parker MD, et al: Genomic and epidemiological analysis of SARS-CoV-2 viruses in Sri Lanka. medRxiv 2021:2021.2005.2005.21256384.

18. Wolfel R, Corman VM, Guggemos W, Seilmaier M, Zange S, Muller MA, Niemeyer D, Jones TC, Vollmar P, Rothe $C$, et al. Virological assessment of hospitalized patients with COVID-2019. Nature. 2020;581(7809):465-9.

19. Widders A, Broom A, Broom J. SARS-CoV-2: The viral shedding vs infectivity dilemma. Infect Dis Health. 2020;25(3):210-5.

20. Coyle PV, Molawi NHA, Kacem MABH, Kahlout RAE, Kuwari EA, Khal AA, Gilliani I, Jeremijenko A, Saeb H, Thani SMA, et al: Inclusion of cycle threshold (CT) values when reporting SARS-CoV-2 RTPCR results improves clinical Interpretation in suspected and confirmed COVID-19. medRxiv 2021:2021.2002.2011.21251557.

21. Kampf G, Lemmen S, Suchomel M: Ct values and infectivity of SARS-CoV-2 on surfaces. The Lancet infectious diseases 2020.

22. Rao SN, Manissero D, Steele VR, Pareja J. A Systematic Review of the Clinical Utility of Cycle Threshold Values in the Context of COVID-19. Infect Dis Ther. 2020;9(3):573-86. 
23. Walsh KA, Spillane S, Comber L, Cardwell K, Harrington P, Connell J, Teljeur C, Broderick N, de Gascun $\mathrm{CF}$, Smith SM, et al. The duration of infectiousness of individuals infected with SARS-CoV-2. J Infect. 2020;81(6):847-56.

24. Watson J, Richter A, Deeks J. Testing for SARS-CoV-2 antibodies. BMJ (Clinical research ed. 2020;370:m3325.

\section{Figures}


RdRP gene

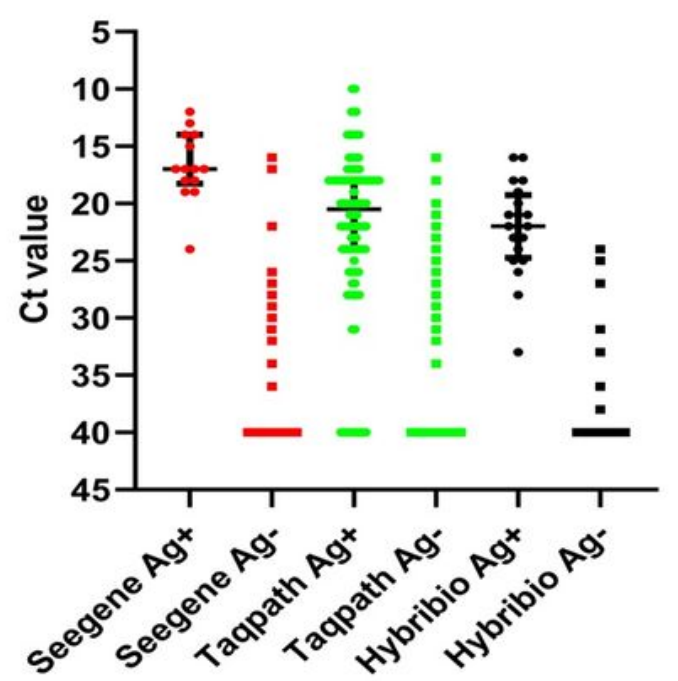

Seegene Vs Taqpath Vs Hybribio Ag+ and Ag-

$\mathrm{N}$ gene

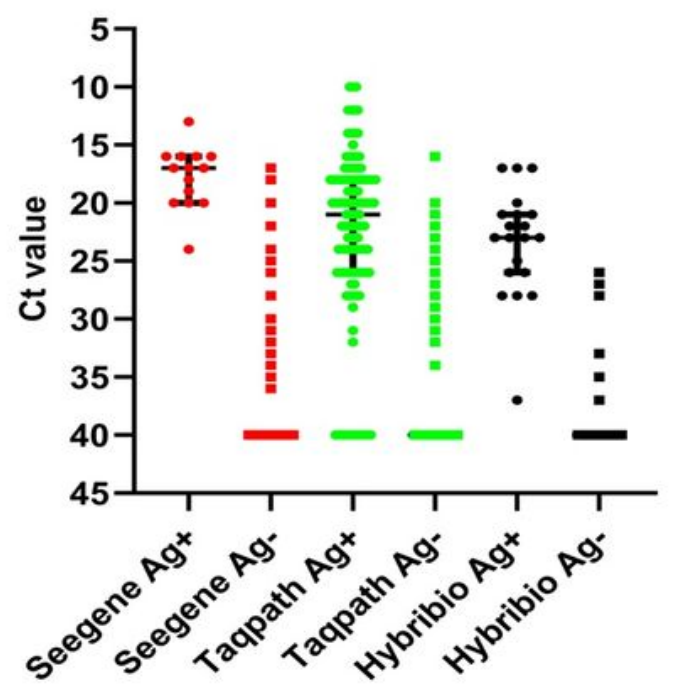

Seegene Vs Taqpath Vs Hybribio Ag+ and Ag-
S gene

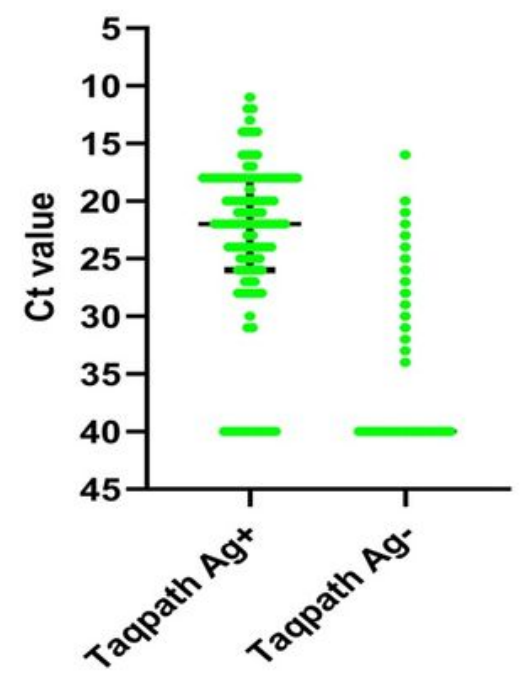

Taqpath Ag+ Vs Taqpath Ag-

E gene

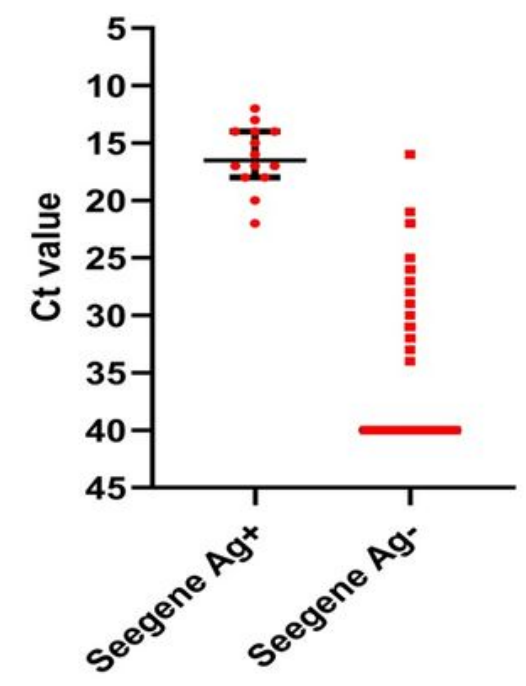

SeegeneAg+ Vs Seegene Ag-

\section{Figure 1}

Cycle threshold $(\mathrm{Ct})$ values for different gene targets in different RT-qPCR kits compared to the results of the SD-Biosensor rapid Ag kit. The Ct values for the RdRP gene (A), S gene (B), N gene (C) and E gene (D) was compared for different kits for Ag positive and Ag negative samples. The lines indicate the median and the error bars the IQR. 
RdRP gene

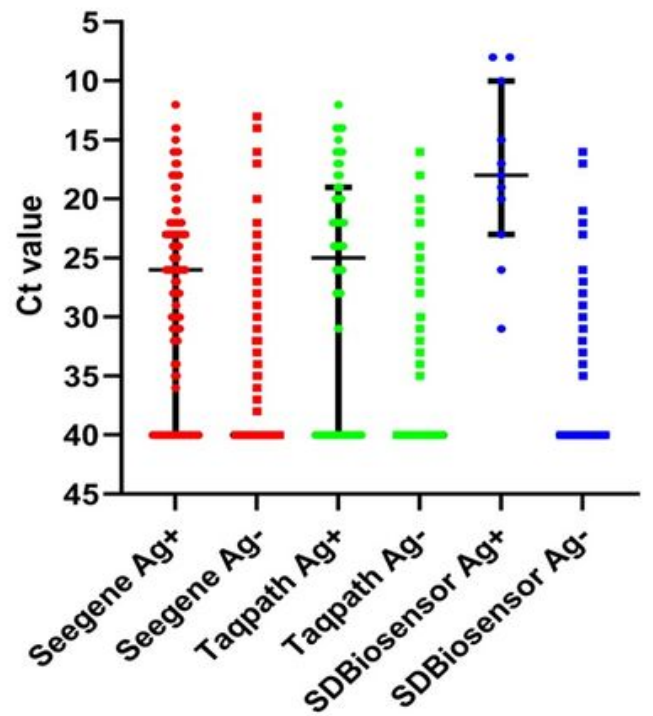

Seegene Vs Taqpath Vs SDBiosensor $\mathrm{Ag}+$ and $\mathrm{Ag}$ -
S gene

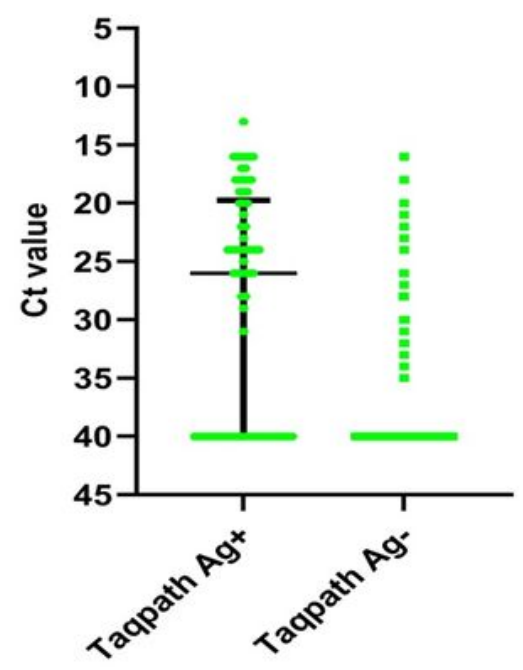

Taqpath Ag+ Vs Taqpath Ag-
N gene

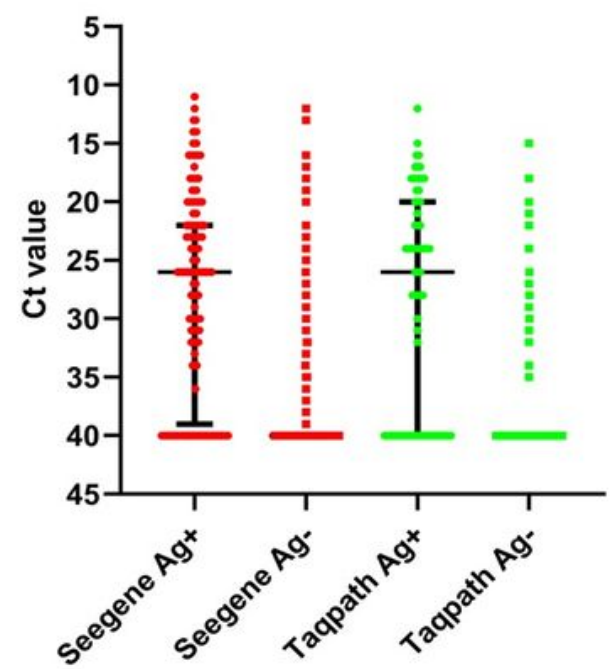

Seegene Vs Taqpath $\mathrm{Ag}+$ and $\mathrm{Ag}$ -
E gene

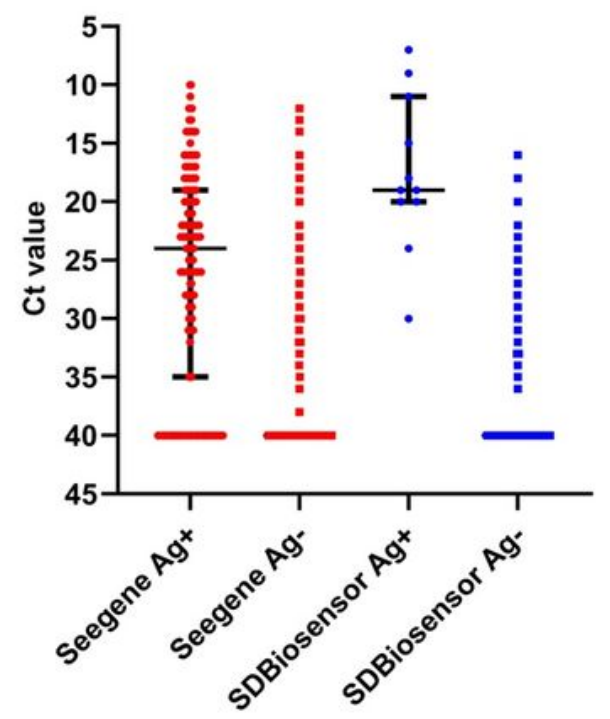

Seegene Vs SDBiosensor Ag+ and Ag-

\section{Figure 2}

Cycle threshold $(\mathrm{Ct})$ values for different gene targets in different RT-qPCR kits compared to the results of the Abbott rapid Ag kit. The $\mathrm{Ct}$ values for the RdRP gene (A), S gene (B), $\mathrm{N}$ gene (C) and E gene (D) was compared for different kits for Ag positive and Ag negative samples. The lines indicate the median and the error bars the IQR. 


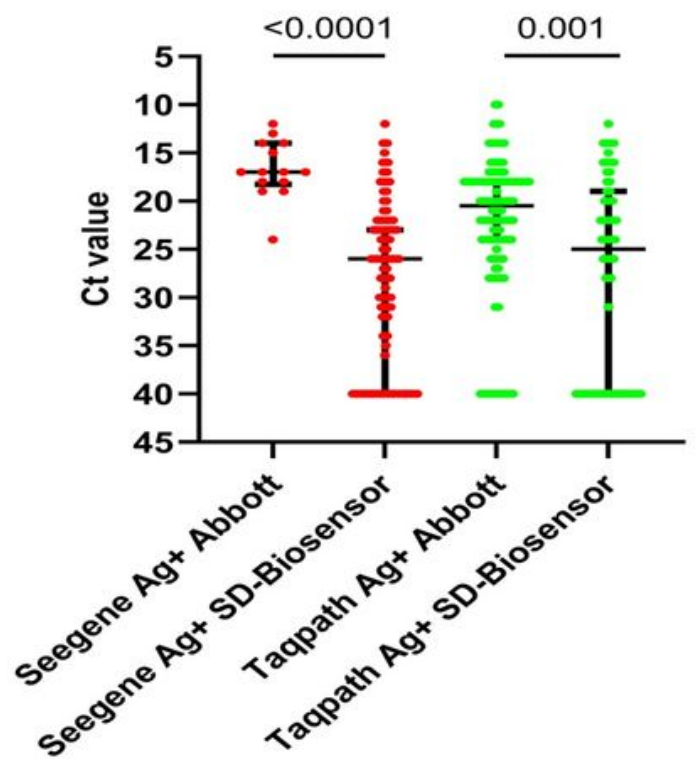

Seegene Vs Taqpath Abbott and SD-Biosensor Ag+

N gene

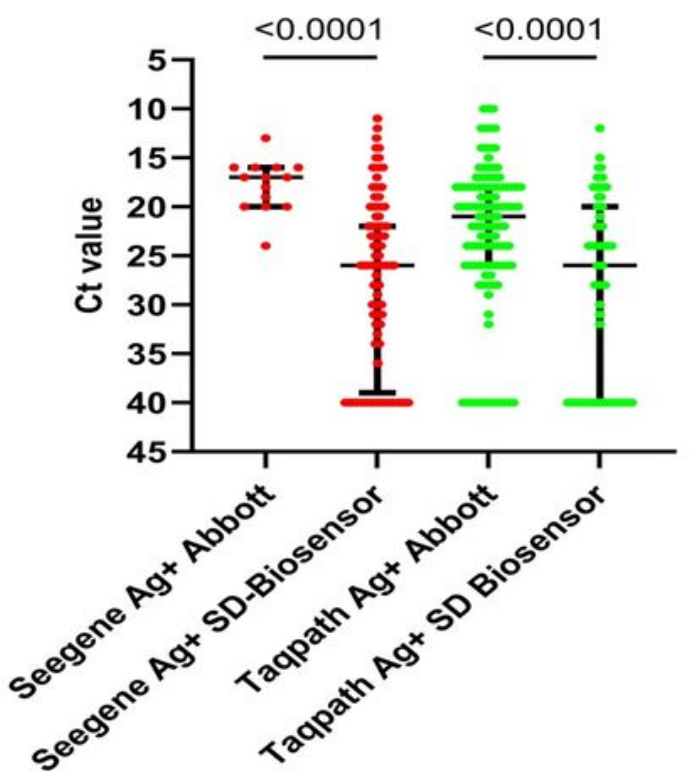

Seegene Vs Taqpath Abbott and SD-Biosensor Ag+

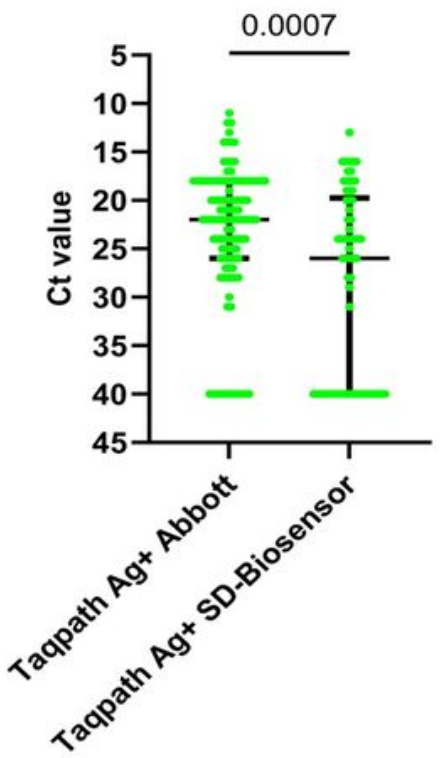

Taqpath (Abbott Ag+ Vs SD-Biosensor

E gene

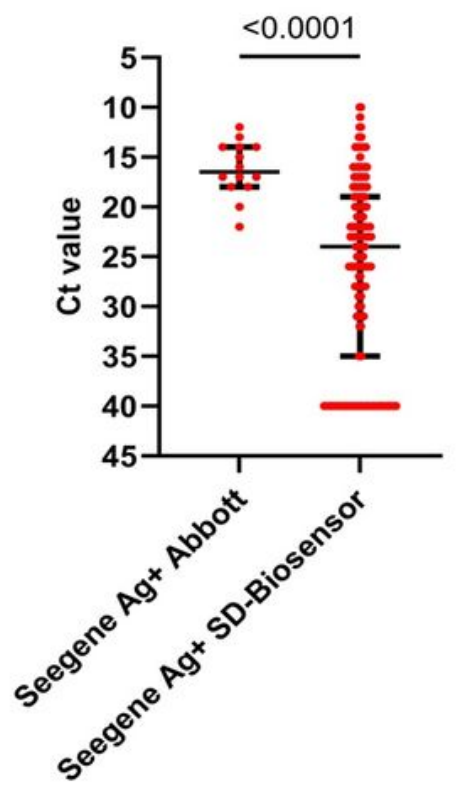

Seegene (Abbott Ag+ Vs SD-Biosensor Ag+)

\section{Figure 3}

Comparison of the Ct values for different gene targets of RT-qPCR kits of positive samples from the SDBiosensor Ag kit and the Abbott Ag kit. The Ct values for the RdRP gene (A), S gene (B), N gene (C) and E gene (D) was compared for different kits for SD-Biosensor Ag positive and Abbot Ag positive samples. The lines indicate the median and the error bars the IQR. 